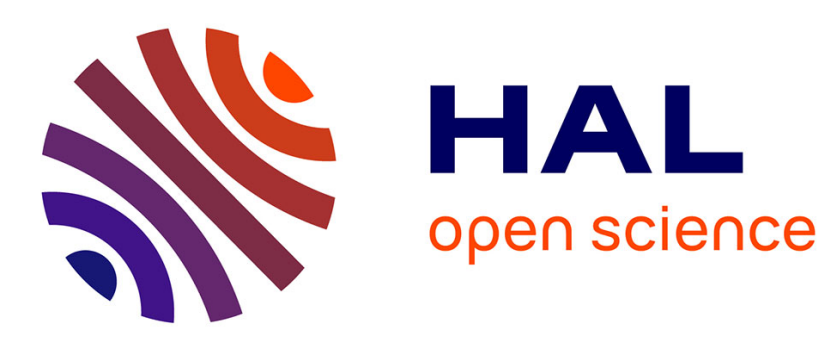

\title{
Modélisation du problème général d'ordonnancement de véhicules sur une ligne de production et d'assemblage \\ Vincent Giard, Jully Jeunet
}

\section{To cite this version:}

Vincent Giard, Jully Jeunet. Modélisation du problème général d'ordonnancement de véhicules sur une ligne de production et d'assemblage. pp.21, 2004. hal-00017757

\section{HAL Id: hal-00017757 \\ https://hal.science/hal-00017757}

Submitted on 25 Jan 2006

HAL is a multi-disciplinary open access archive for the deposit and dissemination of scientific research documents, whether they are published or not. The documents may come from teaching and research institutions in France or abroad, or from public or private research centers.
L'archive ouverte pluridisciplinaire HAL, est destinée au dépôt et à la diffusion de documents scientifiques de niveau recherche, publiés ou non, émanant des établissements d'enseignement et de recherche français ou étrangers, des laboratoires publics ou privés. 


\title{
Modélisation du problème général d'ordonnancement de véhicules sur une ligne de production et d'assemblage
}

\author{
Vincent $_{\text {Giard }}{ }^{1} \&$ Jully Jeunet ${ }^{2}$
}

\begin{abstract}
Résumé
Plusieurs formulations partielles du problème d'ordonnancement sur lignes de production ou d'assemblage dédiées à une production de masse fortement diversifiée - l'industrie automobile constituant l'exemple le plus cité - ont été proposées. Des hypothèses implicites limitent la portée de beaucoup d'entre elles. On proposera ici une formulation générale du problème d'ordonnancement s'appuyant sur des hypothèses réalistes de description du processus physique et prenant en compte l'incidence économique des décisions d'ordonnancement, notamment au niveau de l'appel momentané de renforts et de la prise en compte de l'incidence de rafales sur certains coûts de lancement (atelier de peinture, par exemple). Le modèle linéaire obtenu permet une description explicite et complète de ce problème complexe. La résolution de problèmes réels par la programmation mathématique est difficile en raison de la taille du problème. La description proposée facilite la mise au point d'heuristiques pertinentes.
\end{abstract}

Mots clefs: ligne de production et d'assemblage, ordonnancement sur ligne de produits hétérogènes, contraintes d'espacement, rafales, personnel de renfort, évaluation économique.

\begin{abstract}
Several formulations to the mixed-model assembly line have been proposed in the literature. Such a problem is concerned with the production of a variety of product models. Vehicles fall into that category. The underlying assumptions of those models are often restrictive, therefore limiting their applicability. Our incentive is to develop a general formulation of the car sequencing problem that explicitly takes into account the constraints of the paint shop, those of the body shop and finally the constraints emanating from the assembly shop. The resultant linear program captures the whole complexity of the problem as it is encountered in practice. Solving real-sized instances to optimality would require too much computation time. The proposed description provides a starting point to the development of relevant heuristic solution approaches.
\end{abstract}

Key Words: mixed model assembly line, car sequencing, spacing or contiguity constraints, colour grouping constraints, additional workers, economic evaluation.

1. Lamsade, université Paris Dauphine, \{giard@lamsade.dauphine.fr\}.

2. CNRS, Lamsade, université Paris Dauphine, \{jeunet@lamsade.dauphine.fr\}. 
Modélisation du problème général d'ordonnancement de véhicules sur une ligne de production et d'assemblage

\section{Introduction}

Au début du siècle dernier, les consommateurs étaient largement contraints dans leurs choix de véhicules par l'offre disponible, comme l'illustre la célèbre déclaration d'Henri Ford: le client peut tout choisir, même la couleur, à condition qu'elle soit noire. Les temps ont changé et l'espace de choix en matières d'options n'a désormais plus de limite. Ce problème est maintenant général, la tendance étant de produire en masse des produits personnalisés mais c'est dans l'industrie automobile que la complexité est maximale, ce qui explique que ce secteur soit pris à titre d'exemple. La diversité est obtenue par le montage de modules alternatifs (moteurs de différentes puissances, par exemple) et d'options demandées seulement par certains clients (toit ouvrant, par exemple). Se pose alors le problème du séquencement des véhicules sur la ligne, sachant que la variété conduit, sur certains postes, à des temps opératoires pouvant être supérieurs ou inférieurs au temps de cycle. On est alors confronté au problème qualifié d'ordonnancement sur ligne de produits hétérogènes qui cherche à éviter des phénomènes de désamorçage ou de saturation conduisant à des arrêts de la ligne.

À cet objectif de lissage de la charge de travail, s'ajoute celui du lissage de la consommation de composants non montés systématiquement, en particulier lorsque ceux-ci sont approvisionnés par kanban. La littérature consacrée au traitement de ces deux objectifs est abondante et certaines contributions considèrent conjointement les deux objectifs dans des modèles d'optimisation multicritères.

À ce stade, on doit se rappeler que les usines de production automobile se composent généralement de trois lignes successives sur lesquels les véhicules se succèdent normalement dans le même ordre: la tôlerie, peinture et assemblage. Chaque ligne possède ses propres contraintes. Sur la ligne de peinture, il est économiquement souhaitable d'obtenir un ordonnancement regroupant les véhicules par rafale de teinte, tandis que sur les lignes d'assemblage le respect des contraintes d'espacement permet l'équilibrage de la charge. Ces objectifs ne sont pas faciles à concilier et, dans la littérature, ces contraintes de regroupement des couleurs sont ignorées à quelques rares exceptions près (Giard, 1997, [5] et 2003, [6]). Cet article est sans doute l'un des premiers à considérer simultanément toutes les contraintes dans une formulation optimale du problème de séquencement.

Le $\S 2$ est consacré à une revue des modèles de la littérature relative au problème de l'ordonnancement de produits hétérogènes sur une ligne d'assemblage. Ces modèles sont groupés en trois catégories, en fonction de l'objectif de minimisation poursuivi. Le $\S 3$, page 241 propose une formulation générale du problème d'ordonnancement des véhicules, tel qu'il se rencontre en pratique. Au lieu de modéliser successivement les contraintes provenant des trois ateliers (tôlerie, assemblage et peinture) nous adoptons une différenciation des contraintes fondées sur l'élasticité ou non de la capacité des postes de travail d'où ces contraintes sont issues. Ainsi, les postes capacitaires sont ceux dont la capacité est résolument fixe. La majorité des postes de tôlerie en font partie. Les postes non-capacitaires peuvent voir leur capacité s'accroître en accueillant des travailleurs supplémentaires ou renforts. Nous distinguons un troisième groupe de contraintes relatives à l'atelier de peinture. Le $\S 3$, page 241 conclut en rappelant les apports de la modélisation proposée pour la création de méthodes de résolution exacte et heuristique. 


\section{Revue de la littérature}

Comme nous l'avons souligné en introduction, deux objectifs principaux sont poursuivis dans le problème de séquencement: lisser la charge de travail $(\S 2.1)$, maintenir la consommation de pièces aussi constante que possible (\$2.2, page 238). Dans les systèmes juste-à-temps, le deuxième objectif est primordial tandis que le premier est secondaire. Certains auteurs considèrent ces deux objectifs comme d'égale importance (§2.3, page 239). On terminera par une présentation synthétique des hypothèses implicitement retenues ( $§ 2.4$, page 240$)$, ce qui conduira à la formulation générale que nous proposons.

\subsection{Lissage de la charge}

Cet objectif équivaut également à minimiser les arrêts du convoyeur. L'une de ses formulations est connue sous le nom de problème de la variation du taux de produit. Soit $u_{i}$ le nombre d'unités du modèle $i$ à séquencer, avec $i=1, \ldots, \mathrm{M}$ et soit $\mathrm{N}$, le nombre total de véhicules. On a $\mathrm{N}=\sum_{i=1}^{\mathrm{M}} u_{i}$.

Soit $x_{i j}$ le nombre d'unités de modèle $i$ séquencé entre les positions 1 et $j$. Le taux idéal de produit $i$ dans la séquence de véhicules est $r_{i}=u_{i} / \mathrm{N}$. Entre les positions 1 et $j$, le nombre idéal de modèles $i$ est égal à $j r_{i}$, son nombre réel est $x_{i j}$. L'objectif est donc de minimiser la distance entre le nombre idéal et le nombre réel de chacun des modèles. Ceci s'écrit: $\sum_{j=1}^{\mathrm{N}} \sum_{i=1}^{\mathrm{M}}\left(x_{i j}-j r_{i}\right)^{2}$.

Une autre façon d'aborder le problème est d'imposer des contraintes d'espacement entre modèles identiques de sorte à réguler le rythme de la production. Ces contraintes peuvent être interprétées comme des contraintes d'équilibrage de la charge mais ces formulations ne sont pas nécessairement équivalentes (voir remarque $1 \mathrm{du} \S 3.2 .1 .3$, page 244).

Solution optimale. Mitsumori ([15], 1969) propose une méthode de résolution optimale pour minimiser le risque d'arrêt du convoyeur. Miltenburg et al ([14], 1990) fournissent une formulation du problème par la programmation dynamique qui peut être résolue facilement pour des problèmes de petite taille. Kubiak et Sethi (1991, [11]; 1994, [12]) montrent que le problème peut être formulé comme un problème d'assignation et résolu à l'aide d'algorithmes ad hoc. Xiaobo et Ohno (1994) développent un algorithme de séparation-évaluation (branch and bound) permettant de trouver la solution optimale pour des petites instances. Récemment, Bautista et al. ([1], 2000) ont montré qu'une solution optimale au problème consiste en la répétition d'une sous-séquence optimale. Plus généralement, les auteurs montrent que cette propriété est valable pour n'importe quelle 
Modélisation du problème général d'ordonnancement de véhicules sur une ligne de production et d'assemblage

fonction objectif $\sum_{j=1}^{\mathrm{N}} \sum_{i=1}^{\mathrm{M}} \phi\left(x_{i j}-j r_{i}\right)$ où $\phi$ est une fonction convexe positive possédant un minimum global en 0 . Lorsque ces conditions sont remplies, la solution du problème originel peut être déduite de la solution au problème réduit.

Notons que ces méthodes optimales sont difficilement appropriées pour la résolution de cas réels (entre 500 et 1500 véhicules) pour des raisons de temps de calcul.

Approches heuristiques. Ding et Cheng ([4], 1993) proposent une procédure qui minimise l'écart entre le nombre d'unités produites jusqu'à présent et les quantités idéales pour la période (étape) courante et la période suivante. Korkmazel et Meral ([10], 2001) modifient cette procédure de sorte à considérer davantage de véhicule-candidat dans un éventail plus large de priorités. Les résultats expérimentaux (jusqu'à 15 modèles) montrent la supériorité de cette procédure modifiée. Choi et Shin ([3], 1997) présentent une simple règle de décision pour sélectionner à chaque étape (ou période) le véhicule qui minimise pour chaque option l'écart entre l'espacement courant et la limite spécifiée de cet espacement. Gottlieb et al. ([7], 2003) décrivent plusieurs heuristiques gloutonnes et montrent que leurs versions dynamiques sont plus performantes que leurs versions statiques. Ces heuristiques consistent à choisir de manière itérative le véhicule qui minimise le nombre de violations de contraintes tout en nécessitant le nombre maximum d'options.

Pour des problèmes de taille moyenne (jusqu'à 90 véhicules), Xiaobo et Ohno ([21], 1997 ) mettent en œuvre avec succès un algorithme de recuit simulé. Cet algorithme n'incorpore pas d'éléments spécifiques au problème traité puisqu'une seule règle de transition est utilisée; cette dernière consistant à échanger deux véhicules dans la séquence. Gottlieb et al. ([7], 2003) développent une métaheuristique de type optimisation par colonies de fourmis. Le problème est modélisé comme un graphe dans lequel chaque sommet représente un véhicule et chaque arc $(i, j)$ porte un poids qui mesure la désirabilité du séquencement de $j$ après $i$. Une stratégie élitiste est utilisée pour sélectionner le meilleur véhicule. La performance de cette heuristique est comparée à celle d'un algorithme de seuil (threshold algorithm) utilisant six règles de transition pour la génération de voisinages. Smith et al. ([18], 1996) considèrent explicitement le problème de séquencement avec satisfaction des contraintes d'espacement. Les violations sont prises en compte dans la fonction objectif par l'utilisation d'une matrice de proximité indiquant le niveau de pénalité pour tout couple de véhicules identiques violant la contrainte d'espacement. Un algorithme de descente est appliqué et se retrouve systématiquement coincé dans des optima locaux. Un algorithme de recuit simulé est alors utilisé. Enfin, les approches de type réseaux de neurones d'Hopfield sont adoptées. Les résultats des simulations montrent la supériorité du recuit simulé sur l'algorithme de descente. Les réseaux neuronaux permettent l'obtention de meilleures solutions que celles obtenues par le recuit, à mesure que la taille du problème augmente.

\subsection{Maintien à un niveau constant de la consommation de pièces}

Cet objectif est celui initialement poursuivi par Toyota dans un environnement de juste-à-temps. 
Solution optimale. Bautista et al. ([2], 1996) montrent que le problème est équivalent à la recherche d'un chemin minimum dans un graphe de sorte que n'importe quel algorithme de détermination de chemin extrême peut être utilisé pour obtenir une solution optimale au problème. Les auteurs reconnaissent néanmoins l'inaptitude de ces algorithmes à déterminer la solution optimale pour des problèmles de taille réelle en raison du nombre trop important d'arcs.

Heuristiques. Parmi elles se trouve l'heuristique de Monden, connue sous le nom de Goal Chasing Method ([16], 1983 ou 1998). Sumichrast et al. ([19], 1992) évaluent cette méthode (GCM I) et sa version simplifiée (GCM II) développée chez Toyota. Les auteurs proposent une méthode fondée sur une idée similaire et incluent dans leur expérience de simulation un algorithme proposé par Miltenburg ([14], 1989). Quatre mesures de l'inefficacité de la chaîne sont utilisées: l'incomplétude du travail (le ratio du travail inachevé sur le travail total), l'inactivité (pourcentage du temps durant lequel les travailleurs sont oisifs), le temps passé au poste de travail (pourcentage de temps qu'un travailleur passe à son propre poste de travail; les travailleurs étant autorisés à quitter leur poste pour fournir de l'aide aux postes en congestion), la variance des taux d'utilisation des composants. La procédure développée par les auteurs utilise la même méthode de sélection des véhicules que celle employée dans GCM mais la fonction-objectif est fondée sur la durée totale d'assemblage à tous les postes de travail. Les résultats des simulations montrent la bonne performance de cette procédure. Leu et al. ([13], 1996) développent un algorithme génétique pour minimiser la variabilité de la consommation de pièces. Les auteurs montrent la supériorité de leur algorithme sur l'heuristique de Monden dans la plupart des cas examinés.

\subsection{Optimisation conjointe des deux objectifs précédents}

Solution optimale. Korkmazel et Meral ([10], 2000) traitent le problème de séquencement bi-critère et proposent une formulation en un problème d'assignation (consécutivement aux travaux de Kubiak et Sethi, [12], 1994) qui peut être résolu optimalement pour des cas de petites tailles (jusqu'à 15 véhicules).

Heuristiques. Hyun et al. ([8], 1998) conçoivent un algorithme génétique multiobjectif. Ils considèrent trois objectifs d'égale importance: minimiser l'utilisation de renforts pour faire face aux surcharges de travail, minimiser le coût de lancement, maintenir constant le taux d'utilisation des pièces. La réalisation simultanée de ces trois objectifs n'est pas une tâche évidente dans la mesure où chacun des objectifs peut être conflictuel avec les autres. Les solutions de ces problèmes multi-critères sont des solutions de type optima de Pareto dans lesquelles il est par définition impossible d'améliorer un objectif sans détériorer les autres. L'approche classique de ces problèmes consiste à réduire les différents objectifs en une fonction unique dans laquelle chaque critère occupe un certain poids. Ceci pose le problème de l'affectation des poids ou de manière équivalente, le problème de la définition de l'importance relative de chaque objectif. Les auteurs développent un processus de sélection qui se passe de toute décision subjective. Le processus de sélection fonctionne de la manière suivante. Une solution localisée dans un cube de faible densité possède une plus grande probabilité de survie que toute autre solution située dans un cube de plus forte densité inclus dans la même strate parétienne. 
Modélisation du problème général d'ordonnancement de véhicules sur une ligne de production et d'assemblage

L'algorithme génétique qui en résulte fournit de meilleures solutions que ceux produits par les algorithmes génétiques multi-objectifs traditionnels.

Murata et al. ([17], 996) proposent une méthode d'affectation de poids variables aux différentes fonctions objectif de sorte à rendre la recherche variable. L'idée est d'exploiter plusieurs directions dans la recherche de solutions pareto-optimales.

Tamura et Ohno ([20], 1999) considèrent le problème bi-critère consistant à lisser le taux d'utilisation des composants et la charge de travail à chaque station d'une ligne comportant une ligne principale ainsi qu'une sous-ligne ou brin supplémentaire. L'installation de ce brin se justifie par l'existence de produits nécessitant de longs temps d'assemblage. Trois méthodes de résolution sont proposées et testées sur un nombre limité de cas. La méthode Goal Chasing est adaptée à ce cas de ligne comportant une sous-ligne, un algorithme de recherche «tabou» est mis en œuvre et une approche par la programmation dynamique est développée pour l'obtention de solutions optimales. Les solutions optimales ne peuvent être obtenues que pour des problèmes de petite taille. La méthode «tabou» fournit des solutions satisfaisantes tandis que la méthode Goal Chasing apparaît comme l'algorithme le plus rapide.

\subsection{Hypothèses implicites des approches retenues}

Les différentes approches étudiées peuvent s'analyser à travers plusieurs grilles permettant de mieux cerner la classe de problèmes traités. La première est relative au lissage de la consommation des composants. La seconde concerne la prise en compte possible du coût de lancement lié au changement d'une caractéristique de deux véhicules se suivant dans l'ordonnancement, par exemple un changement de teinte de peinture; on parlera alors de problème de rafales. La troisième est liée à l'incidence de la variabilité de certains temps opératoires, en fonction de caractéristiques des véhicules. Ce point est à l'origine des principales difficultés de l'ordonnancement. Pour des raisons qui seront explicitées, on est amené à distinguer selon que n'est affecté ou non qu'un seul poste de la ligne. Si plusieurs postes sont concernés, la capacité de ces postes peut être intangible - on parlera alors de postes capacitaires - ou non; dans ce dernier cas, une surcharge momentanée de travail peut être absorbée par des renforts.

\begin{tabular}{|c|c|c|c|c|c|}
\hline \multirow{3}{*}{$\begin{array}{l}\text { Modélisation } \\
\text { implicite }\end{array}$} & \multirow{3}{*}{$\begin{array}{l}\text { Prise en compte } \\
\text { du lissage de } \\
\text { consommation } \\
\text { de composants }\end{array}$} & \multirow{3}{*}{$\begin{array}{l}\text { Prise en compte } \\
\text { de problèmes de } \\
\text { rafales }\end{array}$} & \multicolumn{3}{|c|}{$\begin{array}{l}\text { Prise en compte de la variabilité de temps } \\
\text { opératoires sur certains postes }\end{array}$} \\
\hline & & & \multicolumn{2}{|c|}{ Postes non capacitaire } & \multirow{2}{*}{\begin{tabular}{|c|}
$\begin{array}{c}\text { Postes } \\
\text { capacitaires }\end{array}$ \\
$\begin{array}{c}\text { Plusieurs postes } \\
\text { concernés }\end{array}$ \\
\end{tabular}} \\
\hline & & & $\begin{array}{l}\text { Un seul poste } \\
\text { concerné }\end{array}$ & $\begin{array}{c}\text { Plusieurs postes } \\
\text { concernés }\end{array}$ & \\
\hline$\S 2.1$ & Non & Non & $\begin{array}{c}\text { Oui } \\
\left.\text { (implicitement }{ }^{1}\right)\end{array}$ & Non & Non \\
\hline$\S 2.2$ & Oui & Non & Non & Non & Non \\
\hline$\S 3$ & Non & Oui & Oui & Oui & Oui \\
\hline
\end{tabular}

1. Voir remarque $1 \mathrm{du} \S 3.2 .1 .3$, page 244 . 


\section{Formulation du problème d'ordonnancement}

\subsection{Ensemble à ordonnancer}

Soit un ensemble de $\mathrm{N}$ véhicules à ordonnancer. On note $x_{i j}$ une variable binaire valant 1 si le véhicule $i(i=1, \ldots, \mathrm{N})$ a le rang $j$.

Un seul des véhicules $i$ peut avoir le rang $j$, ce que traduit la relation 1 ( $\mathrm{N}$ contraintes):

$$
\sum_{i=1}^{\mathrm{N}} x_{i j}=1, \operatorname{pour} j=1, \ldots, \mathrm{N} \quad \text { relation } 1
$$

Le véhicule $i$ se voit attribuer nécessairement un seul des rangs $j$, ce que traduit la relation 2 ( $\mathrm{N}$ contraintes):

$$
\sum_{j=1}^{\mathrm{N}} x_{i j}=1, \text { pour } i=1, \ldots, \mathrm{N}
$$

relation 2

\subsection{Contraintes relatives à la variabilité de la charge de travail sur certains postes}

La production de masse de produits personnalisés conduit à une différenciation obtenue par un ensemble d'options (toit ouvrant...) pouvant être choisies ou non par le client et l'usage de jeux de modules assurant une même fonction souvent avec des performances techniques et économiques différentes (moteurs...) mais pas toujours (volant à gauche ou à droite, en fonction du client), un véhicule retenant nécessairement un module pour chacun des jeux. Cette personnalisation conduit à une variation de la charge de travail sur certains postes. L'origine de cette variation pouvant être liée à une option ou un module, on parlera de critère (mais les exemples choisis seront liés aux options). Celleci est prise en compte lors de la conception de la ligne sur la base de caractéristiques prévisionnelles moyennes ( $20 \%$ de toits ouvrants, par exemple) et conduit à affecter à ce type de poste une zone plus longue sur le convoyeur ou à prévoir un stock-tampon en aval du poste si celui-ci est fixe. Ces dispositions permettent de synchroniser les postes de la ligne en garantissant que les arrivées s'effectueront bien dans chaque poste de travail à la cadence définie pour la ligne. Il faut alors que l'ordonnancement respecte certaines contraintes que nous allons examiner, faute de quoi le processus sera perturbé par des mécanismes de saturation bloquant progressivement les postes de travail en amont (propagation-amont).

L'analyse du problème conduit à distinguer deux cas de figure. Certains de ces postes affectés par cette variabilité de travail impliquent le respect strict de ces contraintes, généralement en raison de la capacité d'équipements (robots de soudure...); on parle alors de postes capacitaires (qui se trouvent principalement en tôlerie). D'autres postes permettent de s'affranchir de cette contrainte à condition de renforcer le personnel affecté à ce poste, l'outillage utilisé pouvant sans problème s'adapter à cette variation du nombre d'opérateurs; on parle alors de postes non capacitaires. On commencera par analyser le 
Modélisation du problème général d'ordonnancement de véhicules sur une ligne de production et d'assemblage

cas des postes capacitaires (§ 3-2.1). L'analyse des postes non capacitaires en découle immédiatement et conduit à une transformation de contraintes et une modification de la fonction-objectif qui doit prendre en considération le coût additionnel induit par l'éventuelle mobilisation d'opérateurs supplémentaires en raison de l'ordonnancement retenu (§ 3-2.2, page 248).

\section{3-2.1 Prise en compte des contraintes des postes capacitaires}

\subsubsection{Formulation initiale}

On note $k$ l'un des postes capacitaires de la ligne (sous-ensemble $\mathscr{C}_{1}$ de l'ensemble des postes de la ligne). La durée du travail requis par le véhicule $i$ sur le poste de travail $k$ est notée $\theta_{i k}$ et le temps de cycle est noté $\bar{\theta}$, l'arrivée des véhicules sur ce poste étant cadencée par ce temps de cycle.

On note $h$, le rang du véhicule positionné sur le poste de travail $k$ (avec $h=1, \ldots, \mathrm{N})$, le temps de travail à exécuter sur ce véhicule de rang $h$ est $\sum_{i=1}^{\mathrm{N}} \theta_{i k^{x}}{ }_{i h}$.

L'écart entre cette durée et le temps de cycle $\sum_{i=1}^{\mathrm{N}} \theta_{i k^{x}}{ }_{i h}-\bar{\theta}$ correspond à un dépassement s'il est positif et, dans le cas contraire, à une éventuelle possibilité de rattrapage d'une surcharge de travail antérieure.

On note $\mathrm{R}_{k h}$ l'excédent de charge de travail à résorber après traitement du véhicule de rang $h\left(R_{k 0}=0\right)$. Ce report est limité par une quantité de travail $R_{k}^{\max }$, liée au déplacement maximum du véhicule sur le convoyeur ou à la taille des stocks-tampons situés en amont et aval du poste de travail $k$ si le véhicule est traité par un poste fixe ${ }^{1}$. Le fait que ce report maximal $R_{k}^{\max }$ soit fixe est lié à l'hypothèse implicite qu'aucune compensation sur les temps opératoires n'est considérée comme possible avec les postes adjacents ${ }^{2}$.

Ce report $\mathrm{R}_{k h}$, qui ne peut être négatif, intègre la charge de travail non résorbée après traitement du véhicule de rang $h-1$. Une fois traité le véhicule de rang $h$, cet excédent est: $R_{k h}=\operatorname{Max}\left\{0, \operatorname{Min}\left[R_{k, h-1}+\sum_{i=1}^{\mathrm{N}} \theta_{i k^{x}}{ }_{i h}-\bar{\theta}, R_{k}^{\max }\right]\right\}$. Cette contrainte, utilisée de manière récurrente à partir du premier véhicule ordonnancé sur le poste de travail $k$, traduit simplement le fait que la charge cumulée de travail exécuté ne peut dépasser la capacité cumulée de production en tenant compte des contraintes de report maximal. Sa transcription dans une formulation linéaire du problème se fait sans difficulté par l'intermédiaire des relations 3, en utilisant la variable intermédiaire $W_{k h}$.

1. Le stock-amont permet d'attendre la libération du poste fixe et donc d'éviter la saturation. Dans le cas d'un poste embarqué sur convoyeur, ce stock est inutile, l'opérateur se déplaçant. Le stock-aval permet au poste aval de respecter la cadence du cycle et donc évite le désamorçage. Le dimensionnement de ces stocks correspond à l'arrondi supérieur du quotient de l'excédent maximal de travail à résorber (voir ci-après) par le temps de cycle.

2. Dans ce cas, on peut se ramener au cas général en travaillant sur un poste fictif réunissant les postes concernés. 


$$
\begin{array}{r}
W_{k h} \leq R_{k}^{\max } ; W_{k h} \leq R_{k, h-1}+\sum_{\substack{i=1 \\
k \in \mathscr{R}_{1}}}^{\mathrm{N}} \theta_{i k} x_{i h} ; R_{k h} \geq 0 ; R_{k h} \geq W_{k h} \text { pour } h=1, \ldots, \mathrm{N} \text { et } \\
\text { relations } 3
\end{array}
$$

Cette formulation comporte $4 \cdot \mathrm{N}$ contraintes pour chaque poste $k \in \mathscr{K}_{1}$, ce qui fait que l'on a intérêt à traiter des problèmes d'ordonnancement où la taille $\mathrm{N}$ de l'ensemble de véhicules à ordonnancer et le nombre $\mathscr{K}_{1}$ de postes critiques ne sont pas trop conséquents. Si plusieurs postes ont des contraintes «voisines», il suffit alors de ne s'intéresser qu'au plus pénalisant, les autres contraintes étant alors satisfaites. Ce nombre élevé de contraintes pour un poste critique s'explique par la très grande variété postulée de temps opératoires sur ce poste laquelle oblige à vérifier que le temps opératoire de chacun des véhicules ne compromet pas un éventuel rattrapage de retards imputables à un ou plusieurs véhicules de rang inférieur.

\subsubsection{Formulation alternative par les contraintes d'espacement}

Le nombre de contraintes peut diminuer très fortement si le nombre de temps opératoires se limite à 2 que l'on notera $\mathrm{T}_{k_{\overline{-}}}^{\max }$ (présence du critère A, toit ouvrant, par exemple) et $\mathrm{T}_{k}^{\min }$ (absence du critère $\mathrm{A}$, noté $\overline{\mathrm{A}}$ ).

Supposons que le premier véhicule ordonnancé $(h=1)$ possède le critère A $\left(\theta_{i k}=\mathrm{T}_{k}^{\max }\right)$. Lors de la conception de la ligne on a défini le report maximal $R_{k}^{\max }$ comme devant être supérieur ou égal au report $R_{k, 1}=\mathrm{T}_{k}^{\max }-\bar{\theta}$ enregistré après avoir ordonnancé ce premier véhicule.

Le véhicule ordonnancé suivant ne peut posséder le critère A que si le temps résiduel $R_{k}^{\max }-R_{k, 1}=R_{k}^{\max }-\left(\mathrm{T}_{k}^{\max }-\bar{\theta}\right)$ est supérieur à 1 'accroissement de report $\left(\mathrm{T}_{k}^{\max }-\bar{\theta}\right)$. Le nombre maximal $v_{k}$ de véhicules consécutifs «consommant» ce report maximal $R_{k}^{\max }$ est donc l'entier inférieur du quotient $R_{k}^{\max } /\left(\mathrm{T}_{k}^{\max }-\bar{\theta}\right)$. À la conception de la chaîne, on a intérêt donc à définir $R_{k}^{\max }$ comme un multiple de $\left(\mathrm{T}_{k}^{\max }-\bar{\theta}\right)$, ce que nous supposerons par la suite sans perte de généralité. Si les $v_{k}$ premiers véhicules possèdent le critère $A$, le véhicule suivant, de rang $h=v_{k}+1$, ne peut pas posséder ce critère sans violer la contrainte du report maximum. Ce véhicule de rang $v_{k}+1$ permet de diminuer le retard cumulé $R_{k, v_{k}}=R_{k}^{\max }$ après le placement du véhicule de rang $v_{k}$, d'un temps égal à $\left(\bar{\theta}-\mathrm{T}_{k_{\text {min }}}\right)$. Sauf si l'excédent de travail $\left(\mathrm{T}_{k}^{\text {max }}-\bar{\theta}\right)$ du temps opératoire d'un véhicule avec option par rapport au temps de cycle est inférieur au temps inutilisé $\left(\bar{\theta}-\mathrm{T}_{k}^{\text {min }}\right)$ par un véhicule sans option, il faudra plusieurs véhicules sans option avant de pouvoir replacer un véhicule avec option. Ce nombre de véhicules $\mu_{k}$ est égal à la partie entière inférieure du quotient $\left(\mathrm{T}_{k}^{\max }-\bar{\theta}\right) /\left(\bar{\theta}-\mathrm{T}_{k}^{\text {min }}\right)$. Il s'ensuit que $v_{k}$ véhicules consécutifs ayant le critère A sont nécessairement suivis de $\mu_{k}$ véhicules n'ayant pas ce critère. Mais, sur les $v_{k}+\mu_{k}$ premiers véhicules consécutifs, les $v_{k}$ véhicules ayant le critère A peuvent ne pas être consécutifs parce qu'il est évident qu'une telle situation conduit nécessairement le report $R_{k, v_{k}}+\mu_{k}$ du véhicule de rang $v_{k}+\mu_{k}$ à être inférieur 
Modélisation du problème général d'ordonnancement de véhicules sur une ligne de production et d'assemblage

à $R_{k}^{\max }$. Dès lors, si la condition «au plus $v_{k}$ véhicules avec critère sur $v_{k}+\mu_{k}$ véhicules consécutifs» est respectée, les contraintes des relations 3 sont respectées.

Cette démonstration permet de remplacer, pour chaque poste $k \in \mathscr{K}_{1}$, les $4 \cdot \mathrm{N}$ contraintes des relations 3 par les $\left[\mathrm{N}-\left(v_{k}+\mu_{k}\right)+1\right]$ contraintes des relations 4 , qualifiées de contraintes d'espacement. Celles-ci jouent sur des fenêtres glissantes de temps (ou de séquences de véhicules, ce qui revient au même) bornées supérieurement par $h$ et inférieurement par $h-\left(v_{k}+\mu_{k}\right)+1$. Le temps de traitement du véhicule $h$ sur le poste $k$

$$
\sum_{i=1}^{\mathrm{N}} \theta_{i k} x_{i h} \text { est égal à } \mathrm{T}_{k}^{\text {min }} \text { ou à } \mathrm{T}_{k}^{\text {max }} \text { mais, dans la transformation de la formulation, on }
$$
ne s'intéresse plus qu'au fait que les véhicules séquencés possèdent ou non le critère $\mathrm{A}$. Cette information est donnée par $\delta_{i k}$ qui vaut 1 si le véhicule $i$ possède le critère $A$ et 0 , dans le cas contraire. D'une manière générale, un ensemble de $\mathrm{N}$ véhicules caractérisables par $\mathrm{G}$ critères est décrit par une matrice de booléens de terme général $\delta_{i g}$, avec $i=1, \ldots, \mathrm{N}$ et $g=1, \ldots, \mathrm{G}$. Ne s'intéressant ici qu'au critère $\mathrm{A}$, le nombre de véhicules possédant ce critère sur la fenêtre glissante de $\left(v_{k}+\mu_{k}\right)$ véhicules est $\sum_{j=\left\{h-\left(v_{k}+\mu_{k}\right)+1\right\}}^{h} \sum_{i=1}^{\mathrm{N}} \delta_{i k} \cdot x_{i j}$. Comme on l'a vu, ce nombre doit être inférieur à $v_{k}$, ce qui conduit aux relations 4 :

$$
\begin{gathered}
\sum_{j=\left\{h-\left(v_{k}+\mu_{k}\right)+1\right\}}^{h} \sum_{i=1}^{\mathrm{N}} \delta_{i k} \cdot x_{i j} \leq v_{k} \text {, pour } h=v_{k}+\mu_{k}, \ldots, \mathrm{N} \text { et } k \in \mathscr{O}_{1} \text { avec } \\
\mu_{k}=\left(\mathrm{T}_{k}^{\text {max }}-\bar{\theta}\right) /\left(\bar{\theta}-\mathrm{T}_{k}^{\text {min }}\right) \text { et } v_{k}=R_{k}^{\text {max }} /\left(\mathrm{T}_{k}^{\text {max }}-\bar{\theta}\right) \quad \text { relations } 4
\end{gathered}
$$

Ces contraintes d'espacement, utilisées depuis un certain temps dans l'industrie automobile, ont été introduites dans la littérature depuis quelques années (Smith et al., [18], 1996, Giard, [5], 1997; Choi et al., [3], 1997). Leurs justifications et limites par l'analyse fine du processus de production n'ont jamais été apportées à notre connaissance.

\subsubsection{Remarques}

Six remarques peuvent être faites.

- Lissage. Si un seul poste est concerné par cette variabilité, on se retrouve implicitement dans le cas d'un problème de lissage, si la contrainte d'espacement se rapproche de $1 / \mathrm{N}$. Dans le cas contraire, sauf si les modèles sont définis à partir de critères exclusifs mettant en jeu des postes capacitaires (ou non capacitaires) différents, les formulations ne sont pas équivalentes. 
- Contraintes $1 /(v+\mu)$. Dans la pratique, ces relations 4 se retrouve le plus souvent avec $v_{k}=1$; les contraintes d'ordonnancement sont alors du type «1/( $v+\mu) »:$ «pas plus d'un toit ouvrant sur 5 véhicules consécutifs» $\left(\mu_{k}=4\right)$.

- Simplification possible du problème général. Si la variété des temps opératoires sur le poste $k$ est supérieure à deux, il est possible de se ramener à ce cas en retenant comme temps opératoire de chaque critère $f$ de l'ensemble $\mathscr{F}_{k}$ des critères affectant la durée de travail de ce poste $k: \mathrm{T}_{k}^{\max }=\operatorname{Max}_{f}\left\{\theta_{f k}\right\}$ et, $\mathrm{T}_{k}^{\min }=\underset{\left\{f \in \mathscr{F}_{k} \mid \theta_{f k}<\bar{\theta}\right\}}{\operatorname{Max}}\left\{\theta_{f k}<\bar{\theta}\right\}$, dans le cas contraire. Cette simplification permet de limiter la taille du problème (usage des relations 4 au lieu des relations 3 ) mais elle contraint le problème d'ordonnancement plus que nécessaire.

- Ségrégation de flux sur deux brins ligne. Ce type de contrainte peut être utilisé pour gérer la ségrégation des flux lorsqu'une ligne se partage momentanément en deux pour permettre de traiter une quantité de travail plus importante sur un brin que sur l'autre, la ségrégation s'appuyant sur un critère mobilisant des processus techniques différents. Par exemple, en imposant que sur 3 véhicules consécutifs on ait un seul véhicule au plus ayant le critère $A$, on permet de dériver les véhicules ayant cette caractéristique A vers le brin de ligne $\alpha$ et les autres véhicules vers le brin de ligne $\bar{\alpha}$, les deux flux fusionnant ensuite au niveau du poste sur lequel les deux brins convergent, en respectant l'ordre initial. Cette synchronisation implique que la cadence d'entrée sur chacun des postes de travail de l'un des brins soit celle de la ligne (par exemple le brin $\bar{\alpha}$ comportant $\kappa_{\bar{\alpha}}$ postes). Sur l'autre brin $(\alpha)$, le nombre de postes peut être plus faible $\left(\kappa_{\alpha}<\kappa_{\bar{\alpha}}\right)$, pour une charge de travail identique $\left(\bar{\theta} \cdot \kappa_{\bar{\alpha}}\right)$, ce qui permet d'avoir un temps de cycle plus élevé $\left(\theta_{\alpha} \leq \theta \cdot\left(\kappa_{\bar{\alpha}} / \kappa_{\alpha}\right)\right)$ en conservant la synchronisation. des flux ${ }^{1}$. Le non-respect de cette contrainte provoque nécessairement un arrêt de la ligne par saturation, le véhicule ayant le critère A et violant la contrainte étant obligé d'attendre la libération du premier poste du brin de ligne $\alpha$ si celui-ci doit exécuter un travail d'une durée égale

- Limitation des séquences de véhicules ayant le même critère. Ces formulations de contrainte d'espacement peuvent être utilisées pour éviter que l'ordonnancement ne conduise à des séries trop longues de véhicules présentant un même critère ; dans ce cas, ce n'est pas la variabilité des temps opératoires qui justifie la contrainte d'espacement mais des raisons techniques (sur-chauffe...). Par exemple, en imposant que sur 10 véhicules consécutifs on ait un seul véhicule au plus ayant le critère A (critère fictif destiné à limiter la séquence), on limite à 9 la plus longue séquence de véhicules n'ayant pas ce critère.

1. Exemple: $\theta_{\bar{\alpha}}=\theta=1, \kappa_{\bar{\alpha}}=8, \kappa_{\alpha}=2 \Rightarrow \theta_{\alpha} \leq 4$. Le temps de cycle du brin $\alpha$ peut donc être $300 \%$ supérieur à celui du brin $\bar{\alpha}$ (et donc du reste de la ligne). En réalité, c'est le temps de cycle $\theta_{\alpha}$ du brin $\alpha$ qui conditionne l'espacement, le nombre minimal de véhicules de critère $\mathrm{A}$ devant s'intercaler dans l'ordonnancement entre deux véhicules de critère $\mathrm{A}$ étant l'arrondi supérieur du rapport $\left(\theta_{\alpha}-\theta_{\bar{\alpha}}\right) / \theta_{\bar{\alpha}}\left(=\left(\theta_{\alpha}-\theta\right) / \theta\right)$, sachant que ce temps de cycle $\theta_{\alpha}$ et le nombre de postes $\kappa_{\alpha}$ et $\kappa_{\bar{\alpha}}$ concernés par cette scission du flux doivent conduire à une même charge de travail sur chacun des brins et à effectuer des opérations similaires sur les postes suivants. 
Modélisation du problème général d'ordonnancement de véhicules sur une ligne de production et d'assemblage

- Contraintes sur critères croisés. Cette formulation de contrainte d'espacement peut être adaptée pour forcer un véhicule possédant le critère B à être suivi d'au moins $\mu_{\mathrm{BA}}$ véhicules ne possédant pas ce critère $\mathrm{B}$ avant de trouver un véhicule possédant le critère $A$, ces deux critères étant exclusifs ${ }^{1}$. On parle alors de contraintes sur critères croisés, ces contraintes étant utilisées depuis des années dans l'industrie automobile. Elles sont utilisées notamment lorsque le changement de critère amène l'opérateur du poste à changer d'outillage normalement en temps masqué, ce qui n'est possible que si un nombre suffisant de véhicules sépare le véhicule possédant le critère $A$, du véhicule possédant le critère $B$. En général on a $\mu_{B A}=1$. Si la contrainte est symétrique ${ }^{2}$, il faut poser une seconde contrainte. Examinons comment adapter les contraintes des relations 4 en nous intéressant aux véhicules de rang $h>\mu_{\mathrm{BA}}$. Le véhicule de rang $j<h$ ne possède pas le critère $\mathrm{B}$ si

$$
\sum_{i=1}^{\mathrm{N}} \delta_{i \mathrm{~B}} \cdot x_{i j}=0 \text {. Le problème est alors que, sauf spécification contraire, on a le }
$$
droit de faire se succéder des véhicules ayant le critère B si le véhicule de rang $h$ et, le cas échéant, plusieurs véhicules suivants ne possèdent pas le critère A. Pour contourner cette difficulté, il faut introduire la variable indicatrice $y_{h \mathrm{~A}}$ valant 1 si le véhicule de rang $h$ sur le poste $k$ considéré possède le critère $\mathrm{A}$ et 0 dans le cas contraire $y_{h \mathrm{~A}}=\sum_{i=1}^{\mathrm{N}} \delta_{i \mathrm{~A}} \cdot x_{i j}$. L'interdiction recherchée est alors obtenue par les relations 5 .

$$
\begin{aligned}
& \sum_{j=[h-r] i=1}^{h-1} \sum_{i \mathrm{~B}}^{\mathrm{N}} \delta_{i j} \cdot x_{i j}<\left(1-y_{h}\right) \mu_{\mathrm{BA}}, y_{h \mathrm{~A}}=\sum_{i=1}^{\mathrm{N}} \delta_{i \mathrm{~A}} \cdot x_{i j} \\
& \text { pour } h=\mu_{\mathrm{BA}}+1, \ldots, \mathrm{N} ; \mathrm{r}=1, \ldots, \mu_{\mathrm{BA}} \text { et } k \in \mathscr{K}_{1}
\end{aligned}
$$
relations 5

En effet, si le véhicule de rang $h$ possède le critère A, alors $\left(1-y_{h}\right) \mu_{\mathrm{BA}}=0$ et les $\mu_{\mathrm{BA}}$ premières contraintes interdisent la présence de véhicules possédant le critère $\mathrm{B}$ et, dans le cas contraire, $\left(1-y_{h}\right) \mu_{\mathrm{BA}}=\mu_{\mathrm{BA}}$ et les $\mu_{\mathrm{BA}}$ premières contraintes sont toujours satisfaites que les $\mu_{\mathrm{BA}}$ véhicules précédents possèdent ou non le critère B. Si des contraintes d'espacement portent sur le critère B, elles sont exprimées par l'adaptation des relations 4 en remplaçant A par B dans ces relations, et la conjonction de ces contraintes assure le respect des contraintes d'espacement sur B et les contraintes croisées d'espacement de $\mathrm{B}$ par rapport à $\mathrm{A}$ (non symétriques rappelons-le).

1. Exemple: «un véhicule présentant le critère $B$ ne peut suivre un véhicule possédant le critère $\underline{A}$ qu'à condition que 3 véhicules ne possédant pas le_critère $\mathrm{B}$ les séparent»). Les séquences $\mathrm{B}-\mathrm{B}-\mathrm{B}-\mathrm{B}-\mathrm{A}$ et $\mathrm{B}-\mathrm{B}-\mathrm{B}-\mathrm{B}-\mathrm{B}-\mathrm{A}$ sont autorisées mais la séquence $\mathrm{B}-\overline{\mathrm{B}}-\overline{\mathrm{B}}-\mathrm{A}$ est interdite

2. Pour reprendre le même exemple: «un véhicule présentant le critère $A$ ne peut suivre un véhicule possédant le critère $B$ qu'à condition que 3 véhicules ne possédant pas le critère A les séparent». 
- Prise en compte de l'ordonnancement glissant. Le processus de production sur une ligne est continu: le soir on arrête le travail sur la ligne, pour reprendre le lendemain matin le travail là où il s'était arrêté. Pour apprécier l'impact de l'ordonnancement glissant, il faut expliciter de manière plus précise ce qui se passe dans le temps et dans l'espace, d'autant plus que cette mise au point sera indispensable pour l'analyse des renforts ( $\$ 3.2 .2 .2$, page 251). Jusqu'ici, seul le rang d'arrivée d'un véhicule sur le poste $k$ a été pris en considération, le passage du rang au numéro de période de traitement du véhicule s'effectuant immédiatement en raison de la constance du temps de cycle. Ce repérage temporel est «local», le traitement de ce véhicule pouvant se faire le jour du lancement ou le lendemain. En effet, l'ordonnancement décidé pour une journée (période arbitraire utilisée ici pour fixer les idées) ne produit ses effets qu'en partie au cours de la journée. Supposons que la ligne se caractérise par un temps de cycle d'une minute $(\bar{\theta}=1)$, qu'elle comporte $\mathrm{K}=200$ postes ( $\Rightarrow$ charge de travail de 200 minutes) et que le temps quotidien d'ouverture de la ligne soit de $\mathrm{T}=700$ minutes, ce qui conduit à un ordonnancement quotidien de 700 véhicules sur la ligne. En début de journée, au lancement du premier véhicule (première décision mise en œuvre pour le nouvel ordonnancement), les $\mathrm{K}=200$ postes de travail de la ligne sont occupés, le premier par le premier véhicule ordonnancé et les $\mathrm{K}-1=199$ postes suivants par les K -1 derniers véhicules ordonnancés la veille; les $K-1$ derniers véhicules de l'ordonnancement du jour seront donc traités en partie ce jour et en partie le lendemain ${ }^{1}$. L'ordonnancement des $\mathrm{N}$ véhicules pour la nouvelle journée doit nécessairement tenir compte des décisions prises la veille pour respecter correctement les contraintes d'espacement pour chaque critère et les contraintes croisés. Il s'ensuit que pour les contraintes définies par les relations 4 et 5 sont à adapter pour les premiers véhicules à placer. Par exemple, les relations 4 étaient définies pour $h=$ $v_{k}+\mu_{k}, \ldots, N$. En pratique, elles sont définies pour $h=1, \ldots, \mathrm{N}$ mais les $v_{k}+\mu_{k}$ premières contraintes sont liées aux décisions de la veille, lesquelles conduisent à avoir déjà en amont du poste $k$ une séquence de véhicules lancés la veille ${ }^{2}$.

1. Le dernier véhicule des 700 véhicules ordonnancés le jour J passe sur le premier poste de la ligne en fin de cette journée $J$ et passera sur les 199 postes restants au cours de la journée J + 1. Le véhicule ayant le rang 501 est le dernier des véhicules ordonnancé le jour $\mathrm{J}$ et à être traité en totalité à la fin du jour $\mathrm{J}$.

2. Supposons par exemple que $v_{k}=1, \mu_{k}=3$ et que sur les trois derniers véhicules ordonnancés, seul celui du milieu possède le critère A. Dans ces conditions, on $\mathrm{a}$ :

- pour $h=1: 0+1+0+\sum_{i=1}^{\mathrm{N}} \delta_{i \mathrm{~A}} \cdot x_{i 1}<1 \Rightarrow \delta_{i_{f} \mathrm{~A}} \cdot x_{i_{f} 1}=0 \Rightarrow$ interdiction de commencer l'ordonnancement par un véhicule possédant le critère $\mathrm{A}$.

- pour $h=2: 1+0+\sum_{j=1}^{2} \sum_{i=1}^{\mathrm{N}} \delta_{i \mathrm{~A}} \cdot x_{i j}<1 \Rightarrow \delta_{i_{f} \mathrm{~A}} \cdot x_{i_{f} 2}=0 \Rightarrow$ le deuxième véhicule ne peut également pas posséder le critère A.

- pour $h=3: 0+\sum_{j=1}^{3} \sum_{i=1}^{\mathrm{N}} \delta_{i \mathrm{~A}} \cdot x_{i j}<1 \Rightarrow$ le troisième véhicule peut ou non posséder le critère A.

- pour $h>3$ on repart sur la relation générale: $\left(\sum_{j=h-3}^{h} \sum_{i=1}^{\mathrm{N}} \delta_{i \mathrm{~A}} \cdot x_{i j}<1\right)$. 
Modélisation du problème général d'ordonnancement de véhicules sur une ligne de production et d'assemblage

\section{3-2.2 Prise en compte des contraintes des postes non capacitaires}

Comme on l'a indiqué, les postes non capacitaires $\left(k \in \mathscr{K}_{2}\right)$ sont soumis aux mêmes contraintes que les postes capacitaires à la différence près que ces contraintes peuvent être violées parce qu'il est possible d'accroître momentanément la capacité du poste de travail pour faire face à la surcharge de travail. Le personnel utilisé en renfort est amené à se déplacer en fonction de la localisation dans le temps et dans l'espace de ces pointes d'activité et des limites de polyvalence qui le caractérise. Il prend alors en charge un nouveau véhicule pendant que le titulaire du poste achève le travail en cours sur le véhicule précédent.

Ces violations ont donc un coût imputable à la fois aux caractéristiques de l'ensemble à ordonnancer mais aussi de la qualité de l'ordonnancement. Il est alors nécessaire d'introduire une fonction économique d'évaluation permettant de qualifier les ordonnancements possibles. Le problème passe alors de la définition d'un ordonnancement admissible, à celui d'un ordonnancement admissible minimisant une fonction de coût. L'introduction d'une fonction-objectif dans la formalisation du problème se fait à ce stade de la formalisation générale du problème. Elle sera complétée ensuite avec la prise en compte de l'incidence de l'ordonnancement sur d'autres coûts (§ 3.3, page 254). On commencera par décrire la transformation des contraintes $(\S 3.2 .2 .1)$ avant de déterminer le nombre de renforts découlant de l'ordonnancement (\$3.2.2.2, page 251) et d'en tirer les conséquences sur la fonction économique ( $\$ 3.2 .2 .3$, page 253$)$.

\subsubsection{Adaptation des contraintes d'espacement}

Le raisonnement qui a conduit aux relations 4 implique que si les $v_{k}$ premiers véhicules possèdent le critère $A$ et que le véhicule de rang $v_{k}+1$ possède également ce critère, le retard maximal $R_{k}^{\max }$ que ce poste peut absorber est dépassé, l'excédent de charge étant $\mathrm{T}_{k}^{\max }-\bar{\theta}$. A priori, ce dépassement peut être pris en charge par un opérateur en renfort, le titulaire du poste étant occupé par ailleurs, jusqu'au moment où l'excédent est résorbé, ce qui se produit au bout de $\left(\mathrm{T}_{k}^{\max }-\bar{\theta}\right) /\left(\bar{\theta}-\mathrm{T}_{k}^{\text {min }}\right)$ cycles, après quoi le titulaire du poste peut éventuellement prendre la relève du renfort pour achever le travail que celui-ci avait commencé. Pour bien comprendre ce qui se passe physiquement et expliquer les alternatives organisationnelles que l'on a, un exemple est nécessaire.

- Supposons que $\mathrm{T}_{k}^{\text {max }}=80, \mathrm{~T}_{k}^{\text {min }}=50, \bar{\theta}=60$ et $R_{k}^{\text {max }}=40$. Il s'ensuit que $v_{k}=2$ et $\mu_{k}=2$. La surcharge de travail (20) occasionnée par un véhicule ayant le critère A est absorbée par la sous-charge de travail (10) des deux premiers véhicules suivants ne possédant pas le critère A.

- En supposant que les 4 derniers véhicules ordonnancés la veille soient tous sans le critère $A$, la séquence $A-A-\bar{A}-\bar{A}-\bar{A}-\bar{A}$ respecte la contrainte d'espacement et l'évolution de la charge de travail excédentaire à la fin des 6 premiers cycles est $\{20 ; 40 ; 30 ; 20 ; 10 ; 0\}$. À partir du moment où la charge de travail excédentaire retombe à 20 , le véhicule suivant peut à nouveau posséder le critère $\mathrm{A}$, la contrainte «pas plus de deux véhicules possédant le critère A dans une séquence de 4 véhicules» étant respectée, à condition que les deux véhicules suivants ne possèdent 
pas ce critère, autrement dit, la séquence $\mathrm{A}-\mathrm{A}-\overline{\mathrm{A}}-\overline{\mathrm{A}}-\mathrm{A}-\overline{\mathrm{A}}$ est admissible (à condition que le $7 \mathrm{e}$ véhicule ne possède pas le critère $\mathrm{A}$ ).

- Avec la séquence $\mathrm{A}-\mathrm{A}-\mathrm{A}-\overline{\mathrm{A}}-\overline{\mathrm{A}}-\overline{\mathrm{A}}$, l'évolution de la charge de travail excédentaire à la fin des 6 premiers cycles est $\{20 ; 40 ; 60 ; 50 ; 40 ; 30\}$, ce qui nécessite la présence d'un renfort au cours des périodes 3 et 4 avant de retrouver le report maximal admissible. Le Gantt des ressources permet de visualiser deux solutions physiquement possibles.

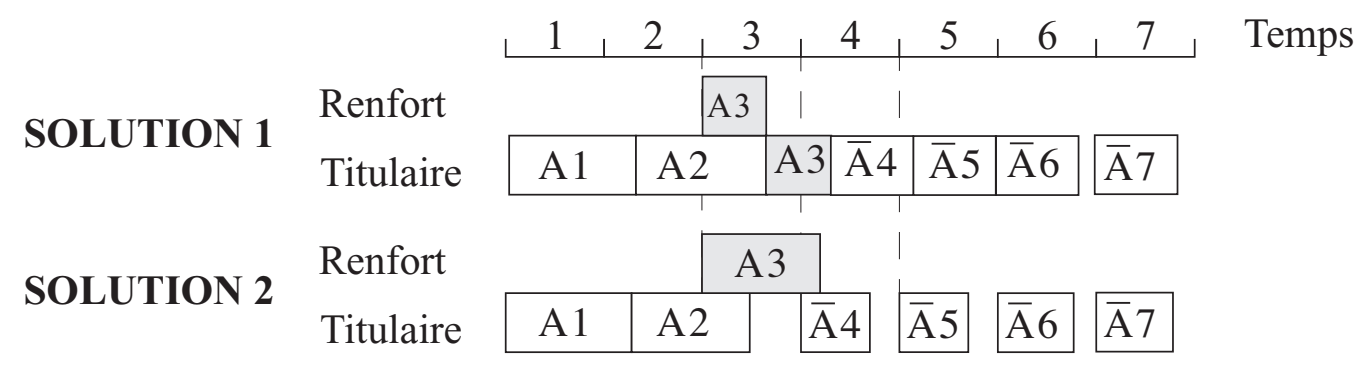

La première solution conduit à un partage du travail à effectuer sur le troisième véhicule et à l'évolution suivante de la charge de travail excédentaire: $\left\{20 ; 40 ; 20^{1}\right.$; $10 ; 0 ; 0\}$. À la fin de la troisième période, tout se passe comme si, pour le choix des véhicules suivants, on recommençait l'ordonnancement sans que les véhicules précédents ( 1 et 2 ) possèdent le critère $A$ (le troisième véhicule possédant ce critère). C'est vrai ici parce que le report maximal n'excède pas le temps opératoire maximal $\left(\mathrm{T}_{k}^{\max } \leq R_{k}^{\max }\right)$. Si cette condition est remplie, toute surcharge se traduit par une réinitialisation des contraintes. Dans le cas contraire, la contrainte d'espacement reste la même.

La seconde solution est sans doute plus réaliste car le partage du travail entre deux opérateurs n'est pas sans problème, en particulier lorsque le temps de cycle est court. De surcroît, elle desserre les contraintes d'ordonnancement comme nous allons le voir. Avec cette solution, l'évolution de la charge de travail excédentaire est: $\{20 ; 40 ; 0 ; 0 ; 0 ; 0\}$. À la fin de la troisième période, tout se passe donc comme si, pour le choix des véhicules suivants, on recommençait l'ordonnancement sans qu'aucun des véhicules ordonnancés avant $(1,2$ et 3$)$ ne possèdent le critère $A$, ce qui est vrai ici parce que le report maximal n'excède pas le temps de cycle $\left(\mathrm{T}_{k}^{\max } \leq \bar{\theta}\right)$. Dans le cas contraire, tout se passe comme si, pour le choix du quatrième véhicule et les suivants, on recommençait l'ordonnancement sans que les véhicules précédents ( 1 et 2 ) possèdent le critère $A$ (le troisième véhicule possédant ce critère).

À partir de ces observations, il est possible d'adapter les contraintes des relations 4 . L'hypothèse organisationnelle que l'on retiendra est celle correspondant à la seconde solution parce qu'elle semble la plus réaliste (sinon, il faut adapter les relations 3). On

1. $80-60=20$ 
Modélisation du problème général d'ordonnancement de véhicules sur une ligne de production et d'assemblage

introduit alors la variable $z_{h k}$ qui vaut 1 si, sur le poste $k$ non capacitaire, on ordonnance en rang $h$ un véhicule possédant le critère A et violant la contrainte du report cumulé maximal. L'impact de cette variable sur la fonction-objectif la conduit à prendre la valeur 0 , sauf en cas de violation de contrainte.

On retiendra pour commencer le cas $\left(\mathrm{T}_{k}^{\max } \leq \bar{\theta}\right)$, qui conduit à une remise à zéro de l'excédent de charge de travail $\mathrm{R}_{k h}$ à résorber après traitement du véhicule de rang $h$ lorsque celui-ci implique la présence d'un renfort. Ceci se traduit, dans la formulation des relations 4 , par une remise à zéro du nombre de véhicules ayant le critère $\mathrm{A}$ $\sum_{j=\left[h-\left(v_{k}+\mu_{k}\right)+1\right]}^{h} \sum_{i=1}^{\mathrm{N}} \delta_{i k} \cdot x_{i j}$, sur la fenêtre glissante de $\left(v_{k}+\mu_{k}\right)$ véhicules de rang inférieur ou égal à $h$. Ce nombre pouvant être remis à zéro, il faut introduire la variable $v_{h k}$ correspondant à ce cumul glissant, ce qui conduit aux relations 6 .

$$
\begin{gathered}
-v_{h k}=\sum_{j=1}^{\left(v_{k}+\mu_{k}\right)} \sum_{i=1}^{\mathrm{N}} \delta_{i k} \cdot x_{i j}, \text { pour } h=v_{k}+\mu_{k} \text { et } k \in \mathscr{J}_{2} \quad \text { relations } 6 \\
-v_{h k}+z_{h k}=v_{(h-1) k}+\sum_{i=1}^{\mathrm{N}} \delta_{i k} \cdot x_{i h}-\sum_{i=1}^{\mathrm{N}} \delta_{i k} \cdot x_{i\left(h-v_{k}+\mu_{k}+1\right)}, \\
\text { pour } h=\left(v_{k}+\mu_{k}\right)+1 \text { et } k \in \mathscr{K}_{2} \text { (relations } 6 \text { - suite) }
\end{gathered}
$$

Dans le premier membre de cette relation, la variable $z_{h k}$ correspond à un dépassement de capacité par mobilisation de renfort si elle est amenée à prendre la valeur 1 .

- $v_{h k}+z_{h k}=v_{(h-1) k} \cdot\left(1-z_{(h-1) k}\right)$

$$
\begin{array}{r}
+\sum_{i=1}^{\mathrm{N}} \delta_{i k} \cdot x_{i h}-\left(\sum_{i=1}^{\mathrm{N}} \delta_{i \mathrm{~A}} \cdot x_{i\left(h-v_{k}+\mu_{k}+1\right)}-s_{h k}\right) \\
\text { avec } s_{h k}=\left(\sum_{i=1}^{\mathrm{N}} \delta_{i k} \cdot x_{i\left(h-v_{k}+\mu_{k}+1\right)} \sum_{j=h-\left(v_{k}+\mu_{k}\right)+1}^{h-1} v_{j k}\right. \\
\text { et } \left.s_{h k} \leq 1 \text { pour } h>\left(v_{k}+\mu_{k}\right)+1 \text { et } k \in \mathscr{R}_{2} \text { (relations } 6-f i n\right)
\end{array}
$$

Le premier membre de cette dernière relation est identique à celui de la relation précédente; justifions les termes du second membre. Le premier correspond au report de la période précédente, s'il est positif. Le second correspond au booléen du critère du véhicule venant d'être choisi au rang $h$ (ce qui ajoute 1 au cumul glissant si ce véhicule possède ce critère). On retranche ensuite le booléen du critère du dernier véhicule de la fenêtre glissante précédente, mais ce dernier terme n'est pas correct si au moins un renfort a été mobilisé pour un véhicule de rang supérieur à celui du dernier véhicule de la fenêtre glissante précédente et donc pour les rangs 
$h-\left(v_{k}+\mu_{k}\right)+1$ à $h-1$. La variable $s_{h k}$ permet de ne retrancher qu'à bon escient le booléen du critère du dernier véhicule de la fenêtre glissante précédente. Dans la définition de cette variable $s_{h k}$, l'expression $\sum_{j=h-\left(v_{k}+\mu_{k}\right)+1}^{h-1} v_{j k}$ correspond au nombre de renforts mobilisés au cours des $\left(v_{k}+\mu_{k}-1\right)$ périodes précédentes; $s_{h k}$ ne peut prendre cette valeur, parce que ce correctif n'intervient pas si le booléen du critère du dernier véhicule de la fenêtre glissante précédente est nul et parce que, dans le cas contraire il ne peut excéder 1; la première observation est prise en compte par la multiplication de ce nombre par ce booléen. La seconde observation est prise en compte par la contrainte $s_{h k} \leq 1$ qui implique que l'on interdise d'avoir deux renforts travaillant simultanément en plus du titulaire du poste, ce qui semble réaliste et assure un certain lissage dans l'utilisation des renforts (lever cette contrainte implique seulement la création d'une nouvelle variable).

Avant d'examiner l'impact économique de l'usage des renforts il convient d'abord de pouvoir les décompter et donc de pouvoir déterminer l'évolution de la charge temporelle des véhicules, ce qui oblige à passer d'une logique locale d'analyse d'un poste, à une logique globale d'analyse simultanée de l'ensemble des postes et implique d'utiliser un même repérage temporel.

\subsubsection{Détermination du nombre de renforts}

L'analyse du problème de l'ordonnancement glissant (page 247) a permis d'introduire la relation entre le rang $h(h=1, \ldots, \mathrm{N})$ d'un véhicule, le poste $k(k=1, \ldots, \mathrm{K})$ sur lequel il se trouve et la date absolue $t(t=1, \ldots, \mathrm{T})$. La demande de renfort pour une journée donnée dépend donc de la position des postes non capacitaires. Le véhicule de rang $h$ dans l'ordonnancement du jour J arrive sur le poste $k$ au début de la période $t=k+h-1$ au cours de la journée $\mathrm{J}$ si $k+h-1 \leq \mathrm{T}$ et le jour $\mathrm{J}+1$, dans le cas contraire. Par exemple, pour $\mathrm{K}=200, \mathrm{~T}=\mathrm{N}=700$, une décision d'ordonnancement du véhicule $h=600$, sur le poste $k=150$ (poste supposé non capacitaire) sera exécutée au cours de la période $t=150$ $+600-1=749$, c'est-à-dire au cours de la 49e minute du jour $\mathrm{J}+1$. Si cette décision implique un renfort, celui-ci n'interviendra que le lendemain de la prise de décision. Certains renforts mobilisés au cours d'une journée auront donc été décidés la veille, tandis que d'autres décidés aujourd'hui ne seront mis en place que le lendemain.

L'opérateur travaillant en renfort est nécessairement amené à partager son temps entre plusieurs postes non capacitaires (dans la pratique, rarement plus d'une demi-douzaine). Ce qui complique l'analyse économique de la décision, c'est que les renforts mobilisés pour une journée sont requis à la suite de décisions prises la veille et d'autres, de décisions prises le jour même ${ }^{1}$. Cet aspect du problème est incontournable car il découle de l'ordonnancement glissant, consistant à générer périodiquement un ordonnancement d'un nouvel ensemble de véhicules, sans attendre que soit complètement traité l'ensemble des véhi-

1. Pour reprendre notre exemple, en $\mathrm{J}+1$, on a un renfort sur le poste $k=150$, à partir de la période 49 , Ce même renfort peut être appelé pour travailler sur le poste non capacitaire $k^{\prime}=100$, à partir de la période 115 , ce qui résulte de l'ordonnancement décidé pour $\mathrm{J}+1$. 
Modélisation du problème général d'ordonnancement de véhicules sur une ligne de production et d'assemblage

cules précédemment ordonnancé. Dans l'analyse économique des renforts, trois positions, au moins, peuvent être adoptées:

- On peut vouloir évaluer l'incidence économique exacte des renforts effectivement mobilisés au cours d'une journée. Cette option conduit à considérer comme des données les conséquences des décisions de renfort prises la veille et exécutées au cours de la journée. Dans cette optique, les décisions de renfort qui ne seront exécutées que le lendemain, ne sont pas prises en compte dans l'évaluation économique.

- L'inconvénient de l'option précédente est de ne pas soumettre à une évaluation économique toutes les décisions prises le jour $\mathrm{J}$ et d'inciter à un report de décisions de renfort vers la fin de l'ordonnancement, là où l'appel à ces renforts n'est pas pénalisé. Ce choix fait donc courir le risque de dégrader l'évaluation de l'ordonnancement du lendemain au profit de celui du jour. C'est pourquoi on peut préférer une formulation hybride dans laquelle l'évaluation économique ajoute au coût des renforts effectivement supportés pour le jour pour lequel l'ordonnancement est établi, la partie du coût des renforts du lendemain résultant de cet ordonnancement. Cet «effet de bord» est d'autant moins marqué que le nombre $\mathrm{N}$ de véhicules à ordonnancer est fort par rapport au nombre total $\mathrm{K}$ de postes.

- Une troisième option est envisageable. Elle consiste à considérer que ce problème est trop compliqué pour être pris en compte dans une formulation globale, d'autant qu'il faut tenir compte dans le calcul des renforts, de leurs déplacements d'un poste à un autre. Dans ces conditions, la minimisation du nombre d'appels de renforts va dans le sens d'une meilleure efficacité économique.

Si aucune de ces positions n'est entièrement satisfaisante, le choix de l'une d'entre elles est nécessaire pour introduire un point de vue économique permettant d'évaluer des alternatives décisionnelles à travers la fonction-objectif. La seconde de ces positions est préférable à l'évidence à la première. Son choix par rapport à la troisième se justifie par le fait que la fonction-objectif devra ultérieurement inclure d'autres éléments de coût, des arbitrages économiques pouvant être à faire entre des purges dans l'atelier de peinture et des renforts. La seconde solution évite l'arbitraire dans la définition des coefficients de la fonction-économique et donne un ordre de grandeur des enjeux économiques liés à l'ordonnancement.

On a vu au $\S 3.2 .1 .2$, page 243, que le véhicule de rang $h$ dans l'ordonnancement du jour $\mathrm{J}$ arrive sur le poste $k$ au début de la période $t=k+h-1$ au cours de la journée $\mathrm{J}$ si $k+h-1 \leq \mathrm{T}$ et le jour $\mathrm{J}+1$, dans le cas contraire. Au début de la période $t$, on traite donc sur le poste $k$ le véhicule de rang $t-k+1$. Compte tenu des hypothèses organisationnelles retenues (solution $2 \mathrm{du}$ Gantt), ce renfort est présent sur un nombre de cycles correspondant à la partie entière inférieure de $\left\{\mathrm{T}_{k}^{\max } / \bar{\theta}\right\}$, que l'on notera $\beta_{k}$. Ce renfort introduit en $t$ sur le poste $k$ non capacitaire $\left(k \in \mathscr{K}_{2}\right)$ est donc présent sur ce poste des périodes $t$ à $t+\beta_{k}-1$, ce qui revient à dire qu'un renfort présent au cours de la période $t$ a commencé son travail au début de $t^{\prime}$ compris entre $t-\beta_{k}+1$ et $t$.

Si l'on ne se préoccupe pas des effets de bord de l'ordonnancement glissant, le cumul du nombre de renforts présents au cours de la période $t$ est donc 
$\sum_{k \in \mathscr{K}_{2} i=1} \sum_{t^{\prime}=t}^{\mathrm{N}} \delta_{i k} \sum_{t-\beta_{k}+1}^{t} x_{i, t-k^{\prime}+1}$, sachant que les hypothèses organisationnelles retenues limitent à 1 ce nombre de renforts à une période quelconque.

On a vu que les véhicules de rang $h$ dans l'ordonnancement arrivent sur le poste $k$ le jour $\mathrm{J}+1$, si $t=k+h-1>\mathrm{T}$. Les éventuels renforts du jour $\mathrm{J}+1$ sont donc pris en compte dans l'expression $\sum_{k \in \mathscr{K} \delta_{2}} \sum_{i=1}^{\mathrm{N}} \delta_{i k} \sum_{t^{\prime}=t-\beta_{k}+1}^{t} x_{i, t-k^{\prime}+1}$. Par contre, cette expression ne tient pas compte de ceux décidés la veille sur le poste $k$ et qui obéissent à la même condition $k+h-1>\mathrm{T}$. On notera $\gamma_{k t}$ ces renforts décidés la veille.

Si l'on suppose que la journée est partagée en deux équipes travaillant chacune pendant $\mathrm{T} / 2$ minutes et s'il est vraisemblable que tout travail de renfort commencé la veille est achevé la veille, le nombre de renforts $z_{1}, z_{2}$ de chacune de deux demi-journées du jour $\mathrm{J}$, dont les valeurs auront tendance à être les plus basses possibles à cause de la fonctionobjectif, est donc tel que:

$$
\begin{aligned}
& z_{1} \geq \sum_{k \in \mathscr{K}_{2} i=1} \sum_{t^{\prime}=t-\beta_{k}+1>0}^{\mathrm{N}} \delta_{i k} \sum_{i, t-k^{\prime}+1}^{t}+\gamma_{k t}, \text { pour } t=1, \ldots, \mathrm{T} / 2 \\
& z_{2} \geq \sum_{k \in \mathscr{K}_{2} i=1} \sum_{t^{\prime}=t-\beta_{k}+1}^{\mathrm{N}} \delta_{i k} \sum_{i, t-k^{\prime}+1}^{t}+\gamma_{k t}, \text { pour } t=\mathrm{T} / 2+1, \ldots, \mathrm{T} \quad \text { relations } 7
\end{aligned}
$$

Le jour $\mathrm{J}+1$, des renforts découleront de l'ordonnancement décidé le jour J. On a vu que ceux-ci découlaient des décisions prises pour les véhicules de rang $h$ sur le poste $k$ pour $k+h-1>\mathrm{T}$. On peut alors reprendre la relation précédente pour des indices de période $t>\mathrm{T}$ :

$$
\begin{aligned}
& z_{3} \geq \sum_{k \in \mathscr{K}_{2}} \sum_{h=1}^{\mathrm{N}} \sum_{i=1}^{\mathrm{N}} \delta_{i k} \sum_{t^{\prime}=t-\beta_{k}+1>0}^{t} x_{i, t-k^{\prime}+1}, \text { pour } t=\mathrm{T}+1, \ldots, \mathrm{T}+\mathrm{K}, \\
& \text { avec } k+h-1 \leq \mathrm{T}
\end{aligned}
$$

On notera que, dans la fonction-objectif que nous allons introduire, les temps de transport d'un poste non capacitaire à un autre ne sont pas pris en compte. Celle-ci est formellement possible avec une matrice de temps de transport d'un poste non capacitaire à un autre mais elle complique la formulation en obligeant à assigner les opérateurs en renfort aux différents renforts (formulation dérivée de formulations classiques).

\subsubsection{Introduction de la fonction économique}

La fonction-objectif correspond au coût des renforts. Pour un coût unitaire de renfort $\gamma$, la fonction-objectif est 
Modélisation du problème général d'ordonnancement de véhicules sur une ligne de production et d'assemblage

$$
\operatorname{Min}\left[\gamma_{1}\left(z_{1}+z_{2}+z_{3}\right)\right]
$$

relation 9

\subsection{Prise en compte des lots de peinture}

Les véhicules passent tous par un atelier de peinture. Le changement de couleur dans l'atelier implique une purge des tuyaux et des pistolets, ce qui a un coût $\gamma_{2}$. Le coût d'une purge peut être ou non dépendant de la couleur précédente. Il semblerait que dans l'industrie automobile, l'hypothèse d'indépendance soit suffisante. Par ailleurs, des contraintes techniques d'encrassement conduisent la taille d'une séquence de véhicules ayant la même couleur (on parle encore de rafale de teinte) à ne pas dépasser un seuil $\mathrm{P}$. On se restreindra ici à un seul poste de peinture mais la généralisation est immédiate et peut concerner d'autres postes pour lesquels un changement fréquent d'outillages ou de réglages génère des coûts que l'on désire limiter.

Notons $u_{h}$ la variable dichotomique valant 1 si le véhicule de rang $h$ a une teinte différente de celle du véhicule précédent $(h-1)$. Dans ces conditions, la fonction- objectif de la relation 10 doit inclure le coût des purges et devient:

$$
\operatorname{Min}\left[\gamma_{1}\left(z_{1}+z_{2}+z_{3}\right)+\gamma_{2} \sum_{h=2}^{N} u_{h}\right]
$$

relation 10

Il faut forcer la variable $u_{h}$ à prendre la valeur 1 lorsqu'il y a changement de réglages sur le poste $k$. Notons $\pi_{i}$ le numéro de teinte utilisé par le véhicule $i$ (avec $\pi_{i}<\mathrm{M}$ ). Le numéro de teinte prise par le véhicule de rang $h$ dans l'ordonnancement est $\sum_{i=1}^{\mathrm{N}} \pi_{i} \cdot x_{i h}$. Le changement de réglage entre le véhicule $h-1$ et le véhicule $h$ arrive lorsque la différence $\sum_{i=1}^{\mathrm{N}} \pi_{i} \cdot x_{i h}-\sum_{i=1}^{\mathrm{N}} \pi_{i} \cdot x_{i(h-1)}$ n'est pas nulle. Pour forcer $u_{h}$ à prendre la valeur 1 en cas de changement, il faut introduire la contrainte suivante:

$$
-\mathrm{M} u_{h} \leq \sum_{i=1}^{\mathrm{N}} \pi_{i} \cdot x_{i h}-\sum_{i=1}^{\mathrm{N}} \pi_{i} \cdot x_{i(h-1)} \leq \mathrm{M} u_{h}, \text { pour } h=2, \ldots, \mathrm{N} \quad \text { relation } 11
$$

qui conduit à l'effet recherché parce que la fonction-objectif tend à rendre nuls le plus possible de $u_{h}$ et que les $u_{h}$ de la relation 11 ne peuvent être nuls que si numéro de réglage du poste $s$ ne change pas et que dans les autres cas (second terme strictement positif ou négatif), $u_{h}$ est nécessairement égal à 1 .

Il convient enfin d'ajouter la contrainte glissante relative au nombre maximal de purges qui est donnée par la relation 12. 


$$
\sum_{h=j}^{j+\mathrm{P}-1} u_{h} \geq 1 \text { pour } j=1, \ldots, \mathrm{N}-\mathrm{P}+1
$$

relation 12

\section{Conclusion}

L'abondance de la littérature relative au problème d'ordonnancement de produits hétérogènes sur ligne d'assemblage témoigne de l'intérêt que suscite un tel problème dans un contexte de production d'une diversité sans cesse renouvelée. Un examen plus minutieux de cette littérature en révèle néanmoins les faiblesses qui prennent leur source dans les hypothèses qui sous-tendent ces modélisations et en limitent substantiellement le champ d'application. Le lancement en production implique l'usage de méthodes de séquencement prenant en compte la totalité des points de vue, qu'ils émanent de la tôlerie, de la peinture ou de l'assemblage. La modélisation proposée dans cet article représente à cet égard une avancée pour la compréhension et la résolution de cette classe de problèmes. Elle nous semble constituer un point de départ important pour la construction de méthodes heuristiques, la résolution exacte semblant compromise pour le traitement de problèmes de taille réelle en raison de l'importance des temps de calcul qu'une telle résolution exigerait. L'élaboration d'une approche heuristique est actuellement en cours et produit des résultats prometteurs sur des instances de grande taille.

\section{Bibliographie}

[1] Bautista, J., Companys, R., Corominas, A., «Note on cyclic sequences in the product rate variation problem», European Journal of Operational Research, 2000, 468-477.

[2] Bautista, J., Companys, R., Corominas, A., «Heuristics and exact algorithms for solving the Monden problem», European Journal of Operational Research, 1993, 101-113.

[3] Choi, W., Shin, H., «A real-time sequence control system for the level production of the automobile assembly line», Computers and Industrial Engineering, 1997, 769-772.

[4] Ding, F.-Y., Cheng, L., «A effective mixed-model assembly line sequencing for Just-In-Time production systems», Journal of Operations Management, 1993, 4550 .

[5] Giard, V., Processus productifs et programmation linéaire, Economica, 1997.

[6] Giard, V., Gestion de la production et des flux, 3e éd., Economica, 2003.

[7] Gottlieb, J., Puchta, M., Solnon, C., «A study of greedy, local search and ant colony optimization approaches for car sequencing problems, in Applications of Evolutionary Computing», Lecture Notes in Computer Science, Springer, 2003, 246-257. 
Modélisation du problème général d'ordonnancement de véhicules sur une ligne de production et d'assemblage

[8] Hyun, C. J., Kim, Y., Kim, Y. K., «A genetic algorithm for multiple objective sequencing problems in mixed model assembly line», Computers and Operations Research, 1998, 675-690.

[9] Kim, Y. K., Hyun, C. J., Kim, Y, «Sequencing in mixed model assembly lines : a genetic algorithm approach», Computers and Operations Research, 1996, 11311145 .

[10] Korkmazel, T., Merel, S., «Bicriteria sequencing methods for the mixed-model assembly line in just-in-time production systems», European Journal of Operational Research, 2001, 188-207.

[11] Kubiak, W., Sethi, S., «A note on level schedules for mixed-model assembly lines in just-in-time production systems», Management Science, 1991, 121-122.

[12] Kubiak, W., Sethi, S., «Optimal just-in-time schedules for flexible transfer lines», Journal of Flexible Manufacturing Systems, 1994, 137-154.

[13] Leu, Y., Matheson, L. A., Rees, L. P., «Sequencing mixed model assembly lines with genetic algorithms», Computers and Industrial Engineering, 1996, $1027-$ 1036.

[14] Miltenburg, J., Steiner, G., Yeomans, S., «Dynamic programing algorithm for scheduling mixed-model just-in-time production systems», Mathematical Computing and Modelling, 1990, 57-66.

[15] Mitsumori, S., «Optimal schedule control of conveyor line», IEEE Trans. Auto. Control, 1969, 633-639

[16] Monden, Y., Toyota Production System : An Integrated Approach to Just-In-Time, 3e éd., Engineering \& Management Press, 1998.

[17] Murata, T., Ishibuchi, H., Tanaka, H, «Multi-objective algorithm and its application to flow shop scheduling», Computers and Industrial Engineering, 1996, 957-968.

[18] Smith, K., Palaniswami, M., Krishnamoorthy, N., «Traditional heuristic versus Hopfield neural network approaches to a car sequencing problem», European Journal of Operational Research, 1996, 300-316.

[19] Sumichrast, R. T., Russell, R. S., Taylor, B. W., «A comparative analysis of sequencing procedures for mixed-model assembly lines in a just-in-time production system», International Journal of Production Research, 1992, 199-214.

[20] Tamura, T ., Long, H., Ohno, K., «A sequencing problem to level part usage rates and work loads for a mixed-model assembly line with a bypass subline», International Journal of Production Economics, 1999, 557-564.

[21] Xiaobo, Z., Ohno, K., «Algorithms for sequencing mixed models on an assembly line in a JIT production system », Computers and Industrial Engineering, 1997, 4756. 